\section{(1) \\ CrossMark}

\title{
Baseline characteristics of idiopathic pulmonary fibrosis: analysis from the Australian Idiopathic Pulmonary Fibrosis Registry
}

\author{
Helen E. Jo (1) ${ }^{1,2}$, Ian Glaspole ${ }^{3,4}$, Christopher Grainge ${ }^{5}$, Nicole Goh ${ }^{3,6}$, \\ Peter M.A. Hopkins ${ }^{7}$, Yuben Moodley ${ }^{8}$, Paul N. Reynolds ${ }^{9}$, Sally Chapman ${ }^{9}$, \\ E. Haydn Walters ${ }^{10}$, Christopher Zappala ${ }^{11}$, Heather Allan ${ }^{12}$, Gregory J. Keir ${ }^{13}$, \\ Andrew Hayen ${ }^{14}$, Wendy A. Cooper ${ }^{14}$, Annabelle M. Mahar ${ }^{15}$, Samantha Ellis ${ }^{15}$, \\ Sacha Macansh ${ }^{12}$ and Tamera J. Corte ${ }^{1,2}$ \\ Affiliations: ${ }^{1}$ Dept of Respiratory Medicine, Royal Prince Alfred Hospital, Sydney, Australia. ${ }^{2}$ Faculty of Medicine, \\ University of Sydney, Sydney, Australia. ${ }^{3}$ Dept of Allergy and Respiratory Medicine, The Alfred Hospital, \\ Melbourne, Australia. ${ }^{4}$ Faculty of Medicine, Monash University, Melbourne, Australia. ${ }^{5}$ Dept of Respiratory \\ Medicine, John Hunter Hospital, Newcastle, Australia. ${ }^{6}$ Dept of Respiratory Medicine, Austin Hospital, \\ Heidelberg, Australia. ${ }^{7}$ School of Medicine, University of Queensland, Brisbane, Australia. ${ }^{8}$ Dept of Respiratory \\ Medicine, Fiona Stanley Hospital, Perth, Australia. ${ }^{9}$ Dept of Respiratory Medicine, Royal Adelaide Hospital, \\ Adelaide, Australia. ${ }^{10}$ University of Tasmania, Hobart, Australia. ${ }^{11}$ Dept of Thoracic Medicine, Royal Brisbane \& \\ Women's Hospital, Brisbane, Australia. ${ }^{12}$ Lung Foundation Australia, Brisbane, Australia. ${ }^{13}$ Dept of Respiratory \\ Medicine, Princess Alexandra Hospital, Brisbane, Australia. ${ }^{14}$ Tissue Pathology and Diagnostic Oncology, \\ Royal Prince Alfred Hospital, Sydney, Australia. ${ }^{15}$ Dept of Radiology, The Alfred Hospital, Melbourne, Australia.
}

Correspondence: Tamera Corte, Royal Prince Alfred Hospital, Missenden Road, Camperdown, 2050, Australia. E-mail: tameracortedme.com

@ERSpublications

Data from the Australian IPF registry shows anti-fibrotic therapy and baseline physiology predict survival in IPF http://ow.ly/Ete2305OkU9

Cite this article as: Jo HE, Glaspole I, Grainge C, et al. Baseline characteristics of idiopathic pulmonary fibrosis: analysis from the Australian Idiopathic Pulmonary Fibrosis Registry. Eur Respir J 2017; 49: 1601592 [https://doi.org/10.1183/13993003.01592-2016].

ABSTRACT The prevalence of idiopathic pulmonary fibrosis (IPF), a fatal and progressive lung disease, is estimated at 1.25-63 out of 100000, making large population studies difficult. Recently, the need for large longitudinal registries to study IPF has been recognised.

The Australian IPF Registry (AIPFR) is a national registry collating comprehensive longitudinal data of IPF patients across Australia. We explored the characteristics of this IPF cohort and the effect of demographic and physiological parameters and specific management on mortality.

Participants in the AIPFR ( $n=647$, mean age $70.9 \pm 8.5$ years, $67.7 \%$ male, median follow up 2 years, range 6 months -4.5 years) displayed a wide range of age, disease severity and co-morbidities that is not present in clinical trial cohorts. The cumulative mortality rate in year one, two, three and four was $5 \%, 24 \%, 37 \%$ and $44 \%$ respectively. Baseline lung function (forced vital capacity, diffusing capacity of the lung for carbon monoxide, composite physiological index) and GAP (gender, age, physiology) stage (hazard ratio 4.64, 95\% CI 3.33-6.47, $\mathrm{p}<0.001)$ were strong predictors of mortality. Patients receiving anti-fibrotic medications had better survival (hazard ratio $0.56,95 \%$ CI $0.34-0.92, \mathrm{p}=0.022$ ) than those not on anti-fibrotic medications, independent of underlying disease severity.

The AIPFR provides important insights into the understanding of the natural history and clinical management of IPF.

This article has been amended according to the erratum published in the March 2017 issue of the European Respiratory Journal. This article has supplementary material available from erj.ersjournals.com 


\section{Introduction}

Idiopathic pulmonary fibrosis (IPF) is a progressive interstitial pneumonia of a largely undefined aetiology and a median survival of only $2-5$ years [1]. While recent therapeutic trials have resulted in significant progress in IPF management [2-5], the selective cohorts in such studies and the short study duration mean that many questions remain unanswered about the generalisability of their findings to the wider IPF population.

It is clear that the natural history of IPF is variable [6], but the factors that predict disease behaviour [7-14] remain largely unknown at an individual level. Long-term observational studies in clinically diverse IPF populations are currently lacking, but would facilitate further understanding of IPF disease biology and its currently unpredictable natural history [15].

Preliminary data from the INSIGHTS-IPF registry [16] in Germany shows that while there are significant similarities between real-world and clinical trial cohorts, such as mean age and body mass index (BMI), there are also significant differences, including severity of disease, presence of co-morbidities and diversity of management, because these factors are often tightly controlled by inclusion and exclusion criteria in clinical trials. Clinical trial results, therefore, may not necessarily be generalisable to the broader IPF population.

The Australian IPF Registry (AIPFR) was launched in February 2012 with the ambitious but necessary goal of characterising IPF patients across Australian clinical practice. This longitudinal registry has prospectively gathered comprehensive documentation to explore the clinical environment and long-term outcomes of these patients. We present the baseline findings from our diverse cohort of clinically diagnosed IPF patients, usually with computed tomography (CT) scans and lung function available but not lung tissue pathology, with regards to their demographic profile, co-morbidities and variations in diagnostic and management patterns. We compare these findings with other registries and clinical trial cohorts to highlight clinical and geographic variability and adherence to current guidelines. We also explore the effects of demographic factors, physiological impairment and specific management strategies, including medication use, on survival.

\section{Methods}

\section{Registry design}

The AIPFR is a national collaboration initiated and administered by the Lung Foundation of Australia. It was established in 2012 and utilises philanthropic pharmaceutical funding in the form of unrestricted educational grants and competitive government (National Health and Medical Research Council (NHMRC)) research grants [17].

This is a multi-centre, prospective, observational registry of incident and prevalent IPF patients across Australia. The registry has ethical approval to operate in all states and territories and this analysis has ethical approval from the Sydney Local Health District ethics committee (protocol number X14-0264). A multidisciplinary steering committee compromising pulmonologists from each state and sub-specialist radiologists and histopathologists oversee the registry, which is also linked to a biobank where the blood from a large sub-population of patients is stored.

\section{Registry participants}

The AIPFR has encouraged recruitment via promotional events at national and international meetings as well as direct mailing to members of professional societies. Recruitment is not limited to tertiary referral centres; any pulmonologist working in Australia is able to refer a patient to the registry. All patients with a diagnosis of IPF referred from their treating pulmonologist are included in the AIPFR. There are no specific exclusion criteria, thus reducing selection bias and creating a cohort encompassing the broad spectrum of IPF diagnosed in current specialist physician practice across Australia.

All registry participants are considered at consent to have 'clinical' IPF as diagnosed by their pulmonologists so are included in this baseline analysis. However, each patient's IPF diagnosis is re-evaluated by a multidisciplinary (radiological, histopathological and clinical) panel (MDM) and categorised as 'definite', 'probable', 'possible' or 'inconsistent' with IPF in accordance to the American Thoracic Society/European

Support statement: Lung Foundation Australia has established the Australian IPF Registry with the generous support of a philanthropic family and unrestricted educational grants from the Foundation partners Roche Products, Pty. Limited and Boehringer Ingelheim and Project partner Bristol-Myers Squibb Australia. This study is supported by an NHMRC grant no. APP1066128. Funding information for this article has been deposited with the Open Funder Registry.

Conflict of interest: Disclosures can be found alongside this article at erj.ersjournals.com

Earn CME accreditation by answering questions about this article. You will find these at erj.ersjournals.com/journal/cme 
Respiratory Society/Japanese Respiratory Society/Latin American Thoracic Association (ATS/ERS/JRS/ALAT) guidelines.

\section{Registry baseline data collection}

AIPFR co-ordinators in each state collect baseline data in proforma questionnaires from participants and their referring physicians. These data include demographics, medical history, quality of life and symptoms, as well as objective investigational data collected up to 6 months prior to registry consent (supplement table 1). The most recent high-resolution computed tomography (HRCT) scans and any surgical lung biopsy specimens are also collected.

\section{Registry MDM data collection and the MDM process}

The HRCT digital images are uploaded to a centralised database for review by the AIPFR radiology review panel. Similarly, histopathology slides, where available, are collected and sent directly to the registry histopathology review centres. There are two radiology panels and one pathology panel, with each having three members who independently review and designate a usual interstitial pneumonia classification in accordance with the ATS/ERS/JRS/ALAT guidelines [1]. All data are then reviewed by the single clinical panel of three IPF-expert pulmonologists who assign an IPF Registry MDM diagnosis (Supplement figure 1). A consensus is assumed in any panel when two or more members agree; any dispute is resolved through panel discussion.

\section{Registry follow-up data collection}

Co-ordinators contact patients and physicians every 6 months to gather contemporaneous data. These include questionnaire data, medical progress and current examination findings as well as results of investigations performed (supplement table 1). All examinations and investigations occur as part of each patient's routine care at their physician's discretion, with no additional visits or investigations mandated for the AIPFR.

\section{Data management}

Data collection was paper-based, with subsequent entry to a customised remote access database (Filemaker Pro). At data entry, plausibility checks (data ranges, drop-down options) were performed to enhance data accuracy. All paper records were kept on-site for data verification.

\section{Statistical analysis}

In this prospective national registry, patient recruitment is limited by feasibility, and thus no formal sample size calculation was performed. Descriptive analysis was performed with categorical data displayed as absolute numbers and relative frequencies. Missing data, primarily due to patients failing to answer a question, was not estimated, but was removed from the denominator when calculating relative frequencies. For missing data on questionnaires other than the St George's Respiratory Questionnaire (SGRQ), the

TABLE 1 Baseline characteristics of Australian Idiopathic Pulmonary Fibrosis Registry patients and comparisons to other registries and clinical trial cohorts

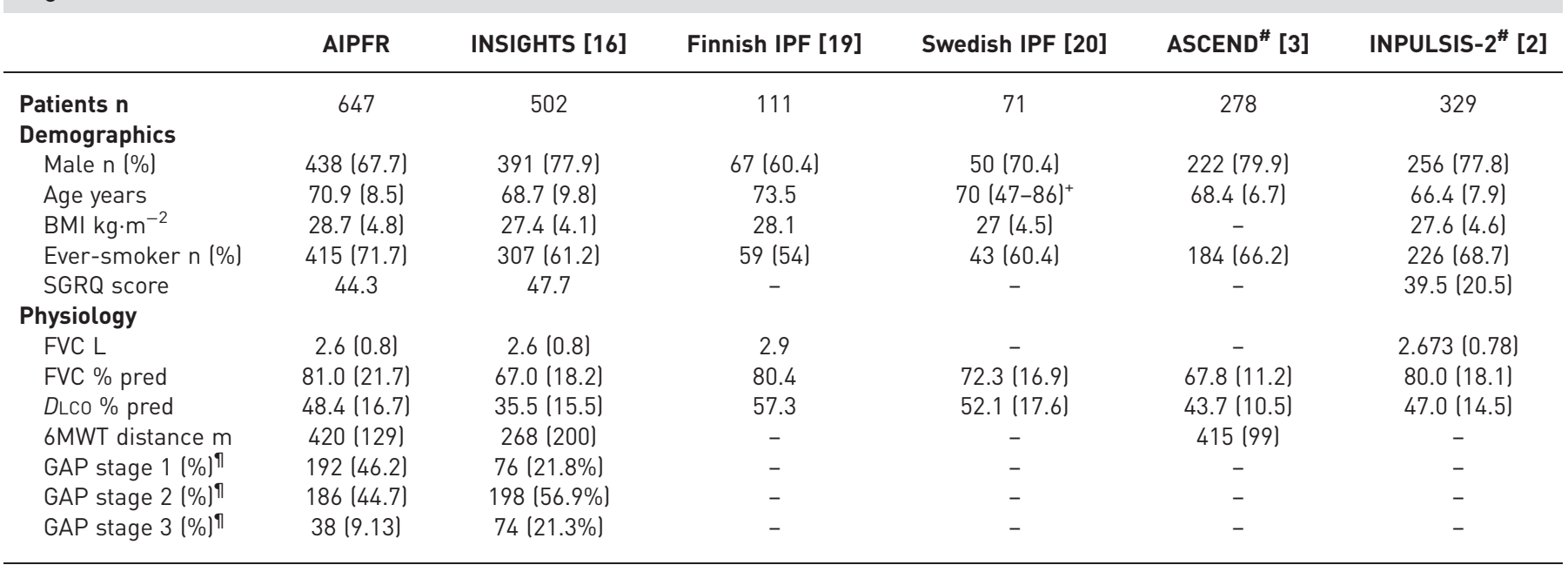

Data are presented as mean (sD) unless otherwise noted. BMI: body mass index; SGRQ: St George's Respiratory Questionnaire; FVC: forced vital capacity; DLCo: diffusion capacity for carbon monoxide; 6MWT: 6-min walk test; GAP: gender, age, physiology. " : treatment group; ": 416 patients had sufficient data for GAP calculation; ${ }^{+}$: data presented as median (range). 

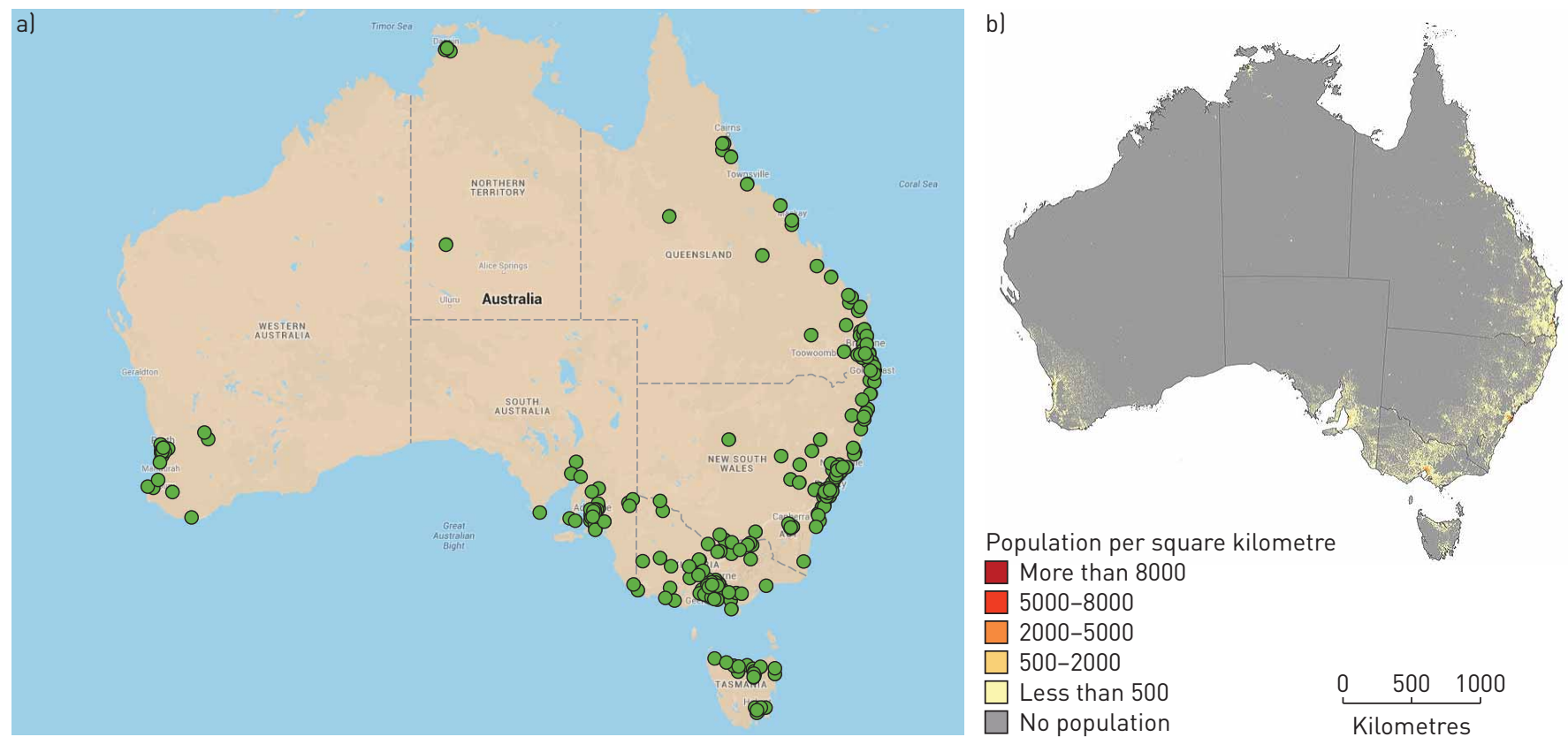

FIGURE 1 Geographical distribution of patients in the Australian Idiopathic Pulmonary Fibrosis Registry (AIPFR). a) The green circles represent the location of patients in the AIPFR. b) Map representing the Australian population distribution (Source: Australian Bureau of Statistics, Cat. No. 1270.0.55.007 - Australian Population Grid, 2011. Published 18 December 2014. ABS data used with permission from the Australian Bureau of Statistics, http://www.abs.gov.au).

mean score of completed questions was used to calculate the total score, provided that $>50 \%$ of the questions were answered. Continuous data are shown as the mean (SD) for normally distributed data or as median (interquartile range) for nonparametric data. Comparisons with other IPF cohorts are descriptive only and based on published data.

Comparisons between groups in the AIPFR were performed using a t-test, Mann-Whitney U test or Chi-squared test as appropriate. The main outcome was mortality, defined as the time from AIPFR baseline until either death or censoring at last date the patient was known to be alive. Patients receiving a lung transplant were censored at that time. Kaplan-Meier estimates and a log-rank test for mortality were performed to calculate mortality per year overall, and by GAP (gender, age, physiology) stage [18]. Univariate and multivariate Cox analysis adjusted for age, sex, BMI and smoking status were performed to assess the impact of baseline variables on mortality.

All statistical analysis were performed using STATA 14.0 (Statacorp, College Station, TX, USA).

\section{Results}

\section{Registry participation}

Although the AIPFR recruits patients throughout the country, the patients primarily reside on the east coast of Australia, reflecting the distribution of the general Australian population (figure 1). Over 170 pulmonologists based in diverse clinical settings, from tertiary centres with dedicated interstitial lung disease clinics to rural sole practices, have referred patients into the AIPFR.

\section{Baseline characteristics: comparison to published IPF cohorts}

At the time of this analysis, there were 647 participants (mean age $70.9 \pm 8.5$ years, $67.7 \%$ male) with a median follow up of 2 years (range 6 months- 4.5 years) enrolled in the AIPFR. The participants' baseline characteristics are outlined in table 1.

Compared to the INSIGHTS-IPF Registry $(n=502)$ [16], the AIPFR participants were older and had a higher BMI and less severe symptom scores on the SGRQ. They also had better baseline lung function than the German cohort, with higher forced vital capacity (FVC) \% predicted, diffusing capacity of the lung for carbon monoxide (DLCO) \% predicted and greater 6-min walk distance. Compared with the Finnish [19] and Swedish [20] IPF registries ( $n=111$ and $n=71$ respectively), however, participants in the AIPFR had lower DLCO, though similar FVC \% predicted.

When compared to clinical trial IPF cohorts such as ASCEND [3] and INPULSIS [2], participants in the AIPFR again were broadly similar. In fact, the mean FVC (L), FVC \% predicted and DLCO $\%$ predicted are 

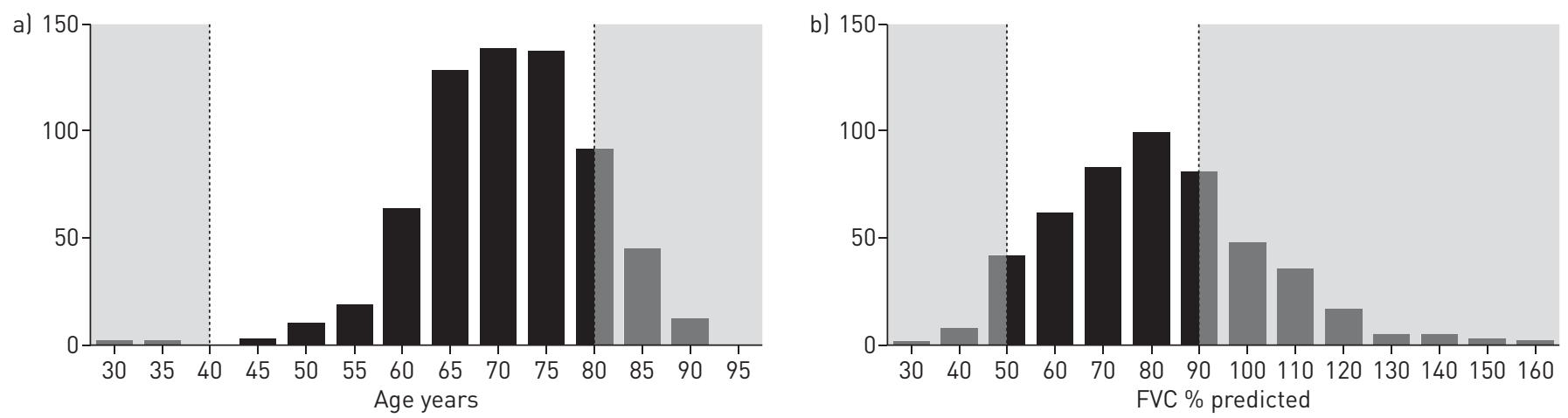

FIGURE 2 Distribution of age and forced vital capacity percentage (FVC) \% predicted in the Australian Idiopathic Pulmonary Fibrosis Registry (AIPFR). a) Age distribution in the AIPFR. b) FVC \% predicted distribution in the AIPFR. The area shaded in grey indicates patients excluded from ASCEND clinical trial inclusion.

almost identical to participants in the INPULSIS cohort, but slightly better than those of the ASCEND cohort. The AIPFR, however, is much more inclusive, with a broader range of ages and disease severity (figure 2).

While significant co-morbidities are often a criterion for exclusion from clinical trials, the presence of co-morbidities is, unsurprisingly, common in clinical IPF, as shown in figure 3. The commonest selfreported co-morbid condition was gastro-oesophageal reflux disease $(\mathrm{n}=233,41 \%)$, followed by pre-existing chronic obstructive pulmonary disease (COPD; $n=197,36 \%)$ and coronary artery disease $(n=161,28 \%)$. Underlying connective tissue disease was also reported in 55 patients (10\%). These were predominantly rheumatoid arthritis $(n=49)$ with smaller numbers reporting scleroderma $(n=2)$, myositis $(n=2)$, Sjögren's $(n=1)$ and systemic lupus erythematosus $(n=1)$. There were also 73 patients $(13 \%)$ who reported a family history of pulmonary fibrosis, one of whom was $<40$ years of age at registry entry.

\section{Investigations and management}

Most AIPFR patients had radiology $(\mathrm{n}=599,93 \%)$ and pulmonary function $(\mathrm{n}=594,92 \%)$ tests performed, while a much smaller number had lung biopsies $(\mathrm{n}=84,13 \%)$. A large number also had autoimmune serology $(\mathrm{n}=393,61 \%)$ and 6 -min walk tests $(\mathrm{n}=281,43 \%)$. Even fewer patients had echocardiography $(\mathrm{n}=213,33 \%)$ or bronchoscopy with or without bronchoalveolar lavage (BAL) $(n=130,20 \%)$. Only a very small number had undergone right heart catheterisation $(n=17,3 \%)$ or cardiopulmonary exercise testing $(n=7,1 \%)$ (figure $4 a)$.

While neither pirfenidone nor nintedanib were available through subsidised government-funded programmes in Australia at this time, 146 patients (23\%) had accessed anti-fibrotics through clinical trials, special access programmes and private purchase.

A considerable number of patients reported treatment with prednisone, either at or prior to registry baseline on their initial questionnaire $(n=251,46 \%)$, with a smaller proportion on azathioprine $(n=38,7 \%)$ and $\mathrm{N}$-acetyl cysteine (NAC) ( $\mathrm{n}=55,11 \%)$ (figure $4 \mathrm{~b})$.

\section{Patient outcomes}

Of the 647 patients with appropriate data, 185 had died and 26 had undergone lung transplantation over a median follow-up of 2 years (range 6 months- 4.5 years) and 1384 total patient years. Figure 5a

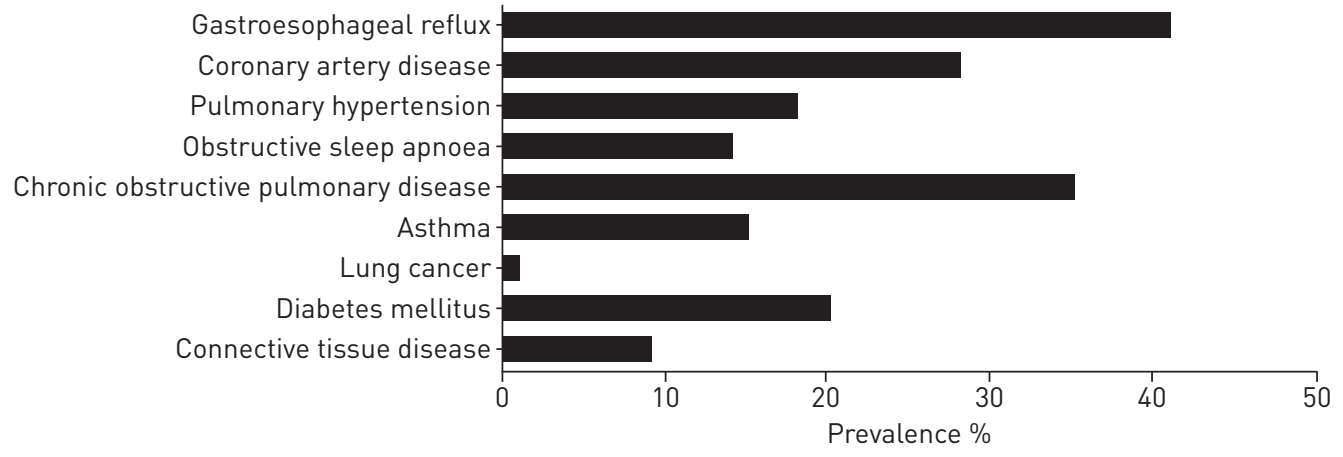

FIGURE 3 Prevalence of co-morbidities in the Australian Idiopathic Pulmonary Fibrosis Registry. 
a)
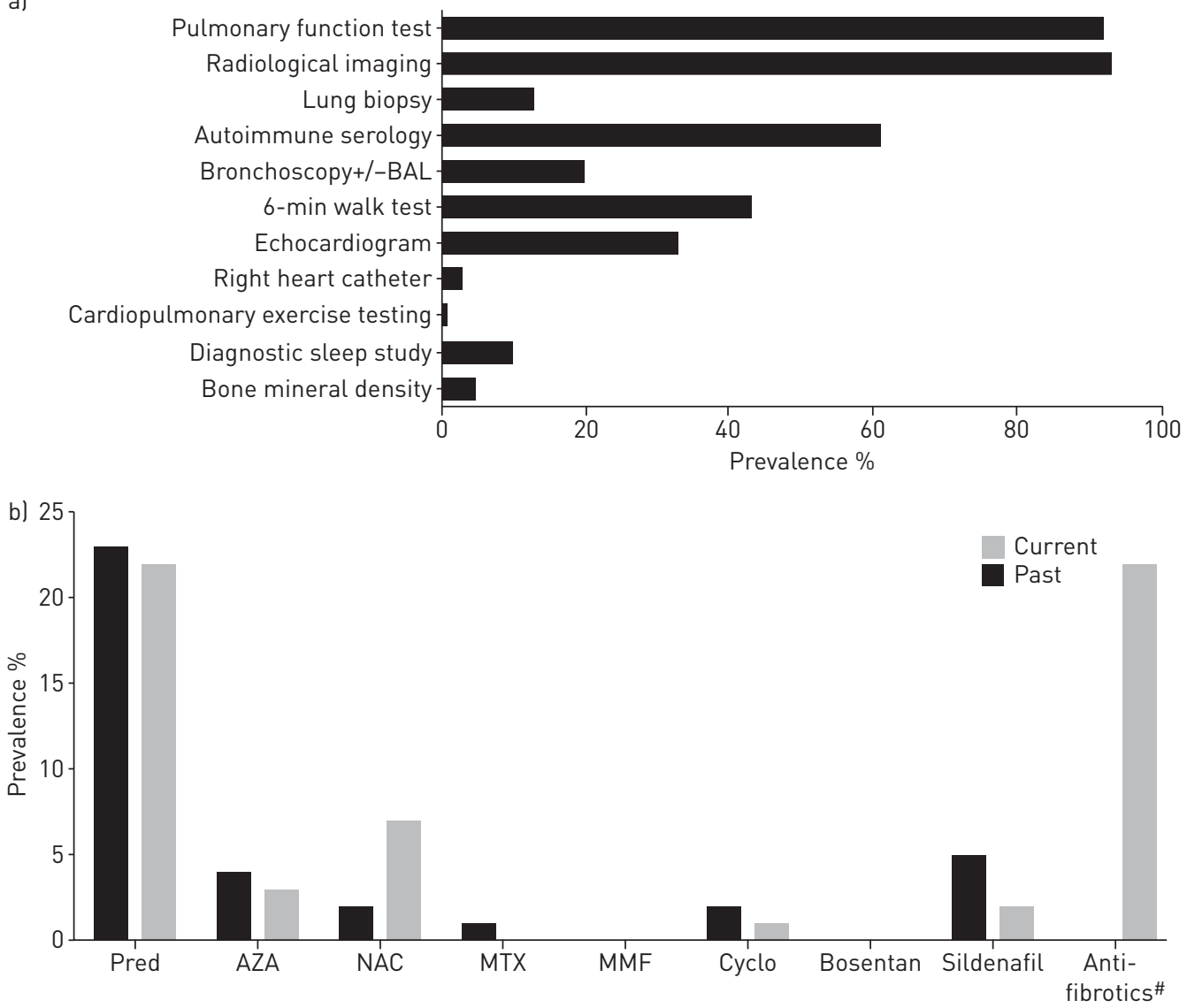

FIGURE 4 a) Frequency of investigations. BAL: bronchiolar lavage. b) Medications at baseline: current and past use. Pred: prednisone; AZA: azathioprine; NAC: N-acetyl cysteine; MTX: methotrexate; MMF: mycophenolate mofetil; Cyclo: cyclophosphamide. "At any time while in the registry.

demonstrates the Kaplan-Meier plot for mortality, showing a cumulative rate of death in year one, two, three and four of $5 \%, 24 \%, 37 \%$ and $44 \%$, respectively.

On univariate Cox analysis of baseline factors, lower BMI and older age were associated with decreased survival. There was no significant association between self-reported co-morbidities and survival. After adjustment for age, gender, BMI and smoking, lower lung function (FVC \% predicted, DLCO \% predicted) was significant for worse mortality, as was a shorter walk distance and greater oxygen desaturation on 6-min walk testing. The composite physiological index (CPI) [21] was also a significant predictor of survival with higher score indicating worse survival (table 2).

In 416 patients with adequate data for calculation of GAP stage at baseline [18], this index was an accurate predictor of mortality, with patients in GAP stage 1 having a 2-year mortality of $6 \%$ compared with $31 \%$ in GAP stage 2 and $66 \%$ in GAP stage 3 (figure $5 \mathrm{~b}$ ).

Patient-reported outcome measures (PROMs), including the total SGRQ score, University of California, San Diego shortness of breath questionnaire (UCSD-SOB), depression score measured on the Hospital Anxiety and Depression Scale (HADS) and cough severity (measured on a visual analogue scale), were all significant predictors of mortality, with higher scores indicating worse symptoms and outcomes. These remained significant even after adjustment for underlying demographic factors (age, gender, BMI and smoking status) and disease severity as assessed by the baseline FVC \% predicted (table 2). The anxiety score, also measured on the HADS, was not a significant predictor of mortality.

\section{Anti-fibrotic therapy}

Patients on anti-fibrotic therapy were younger, more frequently male and able to walk further on 6-min walk testing. They also reported a lower incidence of coronary artery disease. There was, however, no significant difference in the incidence of other co-morbidities or baseline lung function between the two groups (table 3 ). 
FIGURE 5 a) Kaplan-Meier analysis for mortality for the Australian Idiopathic Pulmonary Fibrosis Registry cohort. b) Kaplan-Meier analysis according to GAP stage. GAP: gender, age, physiology. Log rank test $p<0.0001$. c) Kaplan-Meier analysis by use of anti-fibrotic treatment. Log rank test $p<0.0001$.
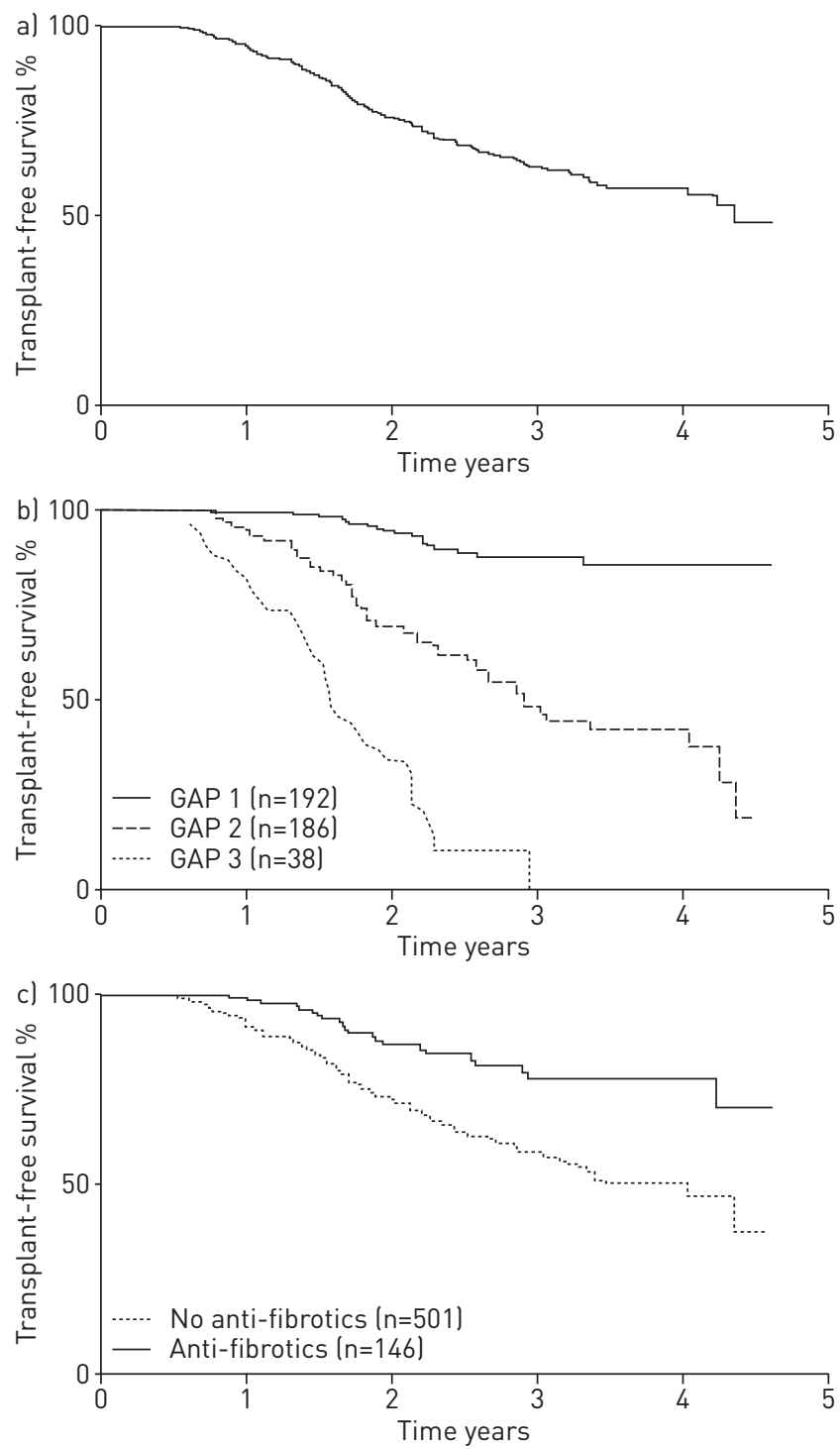

Kaplan-Meier and univariate Cox analysis showed a significant improvement in survival in patients taking anti-fibrotics compared to those not doing so (hazard ratio $0.38,95 \%$ CI $0.24-0.59$, p < 0.001 ) (figure $5 \mathrm{c}$ ). Following multivariate analysis, allowing for age, gender, smoking, BMI and baseline lung function (FVC $\%$ predicted), anti-fibrotic therapy remained a significant predictor of improved survival (hazard ratio 0.56 , 95\% CI 0.34-0.92, $\mathrm{p}=0.022$ ).

\section{Prednisone, azathioprine and NAC}

On Cox analysis, adjusted for baseline age, gender, BMI and smoking, any use of prednisone was associated with increased mortality (hazard ratio 1.59, 95\% CI 1.16-2.19, $\mathrm{p}=0.004$ ). However, neither the use of azathioprine nor NAC was significantly associated with mortality (azathioprine: hazard ratio 1.52, 95\% CI 0.80-2.87, $\mathrm{p}=0.198$; NAC: HR 1.57, 95\% CI 0.92-2.63, $\mathrm{p}=0.084$ ). But after adjustment in the multivariate model for baseline FVC \% predicted, none of prednisone, azathioprine or NAC was significantly associated with mortality.

\section{Discussion}

We report findings from the AIPFR, a large, geographically and clinically diverse national IPF cohort. Unlike many clinical trial cohorts, we demonstrated that AIPFR participants are not homogenous, but have a wide range of age, disease severity and co-morbidities. For the first time from a national IPF registry, we report year one, two, three and four cumulative mortality rates of $5 \%, 24 \%, 37 \%$ and $44 \%$, respectively. We show in our registry cohort that baseline physiology, GAP stage and PROMs are 


\begin{tabular}{|c|c|c|c|c|}
\hline Variable & $\mathbf{n}$ & HR & $95 \% \mathrm{Cl}$ & p-value \\
\hline \multicolumn{5}{|l|}{ Demographics } \\
\hline Age & 643 & 1.02 & $1.00-1.04$ & 0.005 \\
\hline Male & 647 & 1.37 & $0.99-1.90$ & 0.056 \\
\hline BMI & 469 & 0.92 & $0.88-0.96$ & $<0.001$ \\
\hline Smoking & 579 & 1.64 & $1.15-2.35$ & 0.006 \\
\hline \multicolumn{5}{|l|}{ Co-morbidities } \\
\hline Gastro-oesophageal reflux disease & 566 & 1.14 & $0.83-1.55$ & 0.427 \\
\hline Pulmonary hypertension & 529 & 1.31 & $0.89-1.94$ & 0.173 \\
\hline COPD & 552 & 1.33 & $0.97-1.83$ & 0.078 \\
\hline Obstructive sleep apnoea & 562 & 1.12 & $0.73-1.73$ & 0.607 \\
\hline Heart disease & 569 & 1.02 & $0.72-1.43$ & 0.919 \\
\hline Diabetes mellitus & 568 & 0.92 & $0.62-1.34$ & 0.657 \\
\hline Lung cancer & 566 & 0.88 & $0.12-6.29$ & 0.898 \\
\hline \multicolumn{5}{|l|}{ Physiology\# } \\
\hline FVC \% pred" & 465 & 0.67 & $0.61-0.74$ & $<0.001$ \\
\hline DLco \% pred" & 416 & 0.58 & $0.51-0.67$ & $<0.001$ \\
\hline $6 \mathrm{MWT} \mathrm{SpO}_{2}$ at rest & 204 & 0.87 & $0.81-0.94$ & $<0.001$ \\
\hline $6 \mathrm{MWT} \mathrm{SpO}_{2}$ nadir & 173 & 0.94 & $0.91-0.98$ & $<0.001$ \\
\hline 6MWT distance ${ }^{+}$ & 184 & 0.74 & $0.67-0.82$ & $<0.001$ \\
\hline$C P I^{\pi}$ & 412 & 2.17 & $1.80-2.61$ & $<0.001$ \\
\hline GAP stage & 412 & 4.64 & $3.33-6.47$ & $<0.001$ \\
\hline \multicolumn{5}{|l|}{ Patient reported outcome measures } \\
\hline St George's Respiratory questionnaire & 568 & 1.03 & $1.02-1.04$ & $<0.001$ \\
\hline UCSD shortness of breath questionnaire & 574 & 1.03 & $1.02-1.03$ & $<0.001$ \\
\hline Anxiety score & 567 & 1.02 & $0.99-1.06$ & 0.214 \\
\hline Depression score & 567 & 1.11 & $1.07-1.16$ & $<0.001$ \\
\hline Cough severity & 509 & 1.01 & $1.00-1.14$ & 0.030 \\
\hline
\end{tabular}

important prognostic determinants for mortality. Further, we have also demonstrated that anti-fibrotic medication (pirfenidone or nintedanib) are associated with better survival, reflecting the outcomes from clinical trials [2,3] and strengthening support for the current IPF management guidelines [22] in a real-world context.

Unlike most IPF registries in existence [16, 20, 23], AIPFR patient recruitment is not limited to specialised lung centres, allowing greater diversity in recruitment. The AIPFR and other registries [16, 19, 20] highlight the similarities, but also the substantial differences, in real-world IPF compared to clinical trial cohorts [2, 3]. In particular, there has been inclusion of a broader range of age, lung function and co-morbidities not seen in the clinical trial cohorts. While homogenous clinical trial cohorts are important to reduce 'noise', they can lack generalisability.

Using the AIPFR we have been able to confirm that baseline lung function (FVC \% predicted, DLCO \% predicted and CPI) [7, 8, 24-27] and GAP stage [18] are indeed important prognostic markers for mortality. While the questionnaires used in the AIPFR are not specifically designed for IPF, many of these PROMs were also significant predictors of mortality in our IPF cohort. This combination of real-world IPF patients with longitudinal outcome data places the AIPFR in a valuable position to explore disease natural history, and to examine the adoption of and outcomes from new IPF management strategies.

The changing landscape of IPF management is already being reflected in the registry. The AIPFR started recruitment in February 2012, a period in which IPF management was undergoing significant change. Prednisone and other immunosuppressive medications, which had been the conventional approach to treatment $[28,29]$, were shown to be harmful [30] and thus the IPF guidelines in 2011 recommended against their use [1]. Despite this, many patients in the AIPFR were still on these medications, reflecting slow adoption of changing IPF management. Interestingly, we showed that patients who had used prednisone had worse survival than those who had never been on this treatment. This, however, was not 
TABLE 3 Comparison of baseline characteristics between patients by anti-fibrotic therapy use

\begin{tabular}{|c|c|c|c|}
\hline Variable & No anti-fibrotic & Anti-fibrotic & p-value \\
\hline $\mathbf{n}$ & 501 & 146 & \\
\hline \multicolumn{4}{|l|}{ Demographics } \\
\hline Age years (sD) & $71.5(8.9)$ & $68.9(6.8)$ & 0.001 \\
\hline Male & $326(65)$ & 112 (77) & 0.008 \\
\hline Ever smoker & $383(76)$ & 101 (69) & 0.084 \\
\hline $\mathrm{BMI} \mathrm{kg} \cdot \mathrm{m}^{-2}$ (sD) & $28.5(4.82)$ & $29.2(4.71)$ & 0.217 \\
\hline \multicolumn{4}{|l|}{ Physiology } \\
\hline FVC L (SD) & $2.57(0.79)$ & $2.72(0.73)$ & 0.072 \\
\hline FVC \% predicted (SD) & $81.4(23.0)$ & $79.73(17.0)$ & 0.474 \\
\hline DLco \% predicted (SD) & $48.1(17.5)$ & $49.20(14.3)$ & 0.570 \\
\hline 6MWT distance m median (IQR) ${ }^{\#}$ & $404(310-480)$ & $467(410-529)$ & $<0.001$ \\
\hline $6 \mathrm{MWT} \mathrm{SpO}_{2}$ at rest median $(\mathrm{IQR})^{\#}$ & $96 \%(93-97)$ & $96 \%(94-98)$ & 0.063 \\
\hline $6 \mathrm{MWT} \mathrm{SpO}_{2}$ nadir median $(\mathrm{IQR})^{\#}$ & $85 \%(81-89)$ & $87 \%(83-92)$ & 0.164 \\
\hline CPI (sD) & $45.0(14.9)$ & $45.5(11.3)$ & 0.751 \\
\hline \multicolumn{4}{|l|}{ Co-morbidities } \\
\hline GORD & $180(42.2)$ & $53(38.1)$ & 0.402 \\
\hline Pulmonary HT & $76(19.4)$ & $20(14.6)$ & 0.211 \\
\hline COPD & $153(37.1)$ & $44(31.4)$ & 0.223 \\
\hline OSA & $59(14.1)$ & $24(16.9)$ & 0.407 \\
\hline CAD & $134(31.4)$ & 27 (19.0) & 0.005 \\
\hline Diabetes mellitus & $92(21.6)$ & 27 (19.0) & 0.513 \\
\hline
\end{tabular}

Data are presented as $\mathrm{n}(\%)$ unless otherwise stated. Data in bold are statistically significant. BMI: body mass index; FVC: forced vital capacity; DLCO: diffusion capacity for carbon monoxide; 6MWT: 6-min walk test; $\mathrm{SpO}_{2}$ : arterial oxygen saturation measured by pulse oximetry; CPI: composite physiological index; GORD: gastro-oesophageal reflux disease; Pulmonary HT: pulmonary hypertension; COPD: chronic obstructive pulmonary disease; OSA: obstructive sleep apnoea; CAD: coronary artery disease. " : Mann-Whitney U test.

significant when adjusted for baseline FVC \% predicted and thus may reflect underlying disease severity rather than a prednisone effect per se.

The use of anti-fibrotic therapy, although it is not readily available in Australia, is also being reflected in the AIPFR, with 146 patients (23\%) already receiving treatment with nintedanib or pirfenidone. We have demonstrated that patients on anti-fibrotic medications have better survival independent of their baseline disease severity. However, these results must be interpreted with caution because this is a retrospective analysis rather than a randomised controlled trial, and the cases and controls were not fully matched. Those on anti-fibrotic therapy were generally younger and more frequently male, and had better exercise capacity and significantly less self-reported coronary artery disease. However, multivariate analysis adjusting for these, and baseline demographic factors, still showed improved survival in the anti-fibrotic treatment group.

The diversity of recruitment to the AIPFR across Australia has allowed us to explore variation in clinical practice, particularly with regards to the use of diagnostic investigations and adherence to guidelines. Performance of investigations recommended in the guidelines was high (lung function testing, chest imaging), whereas those with less clear evidence (serological testing) were lower. Bronchoscopy (with or without BAL) was relatively uncommon, again reflecting current guideline recommendations against its routine use for evaluating IPF patients. In terms of investigations used for prognostication (6-min walk test, cardiopulmonary exercise testing), use was variable, reflecting the lack of strong evidence or guidelines supporting their use at this time [1].

Our study has several limitations. First, as a real-world database, the information available for each patient was based on individual clinical practice rather than trial protocol. No estimates were made for missing categorical data, but given the large numbers we feel we were able to perform robust statistical analyses. Second, because this was not a clinical trial we cannot make accurate comparisons between different treatment groups. Importantly, while most patients reported starting anti-fibrotic medications at baseline, some patients reported starting while enrolled in the registry. The median time from AIPFR consent to initiation of anti-fibrotic therapy was, however, 6 days prior (interquartile range 3.8 months prior to 5.6 months after). Additionally, start times as well as medication use and co-morbidities are self-reported as part of a structured questionnaire, and their accuracy may be limited. Last, the patients in our study were diagnosed with IPF by their treating pulmonologist. While not all of the patients would fit the ATS/ 
ERS/JRS/ALAT guideline criteria for IPF diagnosis, the registry is purposefully broad in its recruitment to ensure diversity not seen in clinical trials. Indeed, all participants' IPF diagnoses are reviewed by a registry MDM panel to assess adherence to IPF diagnostic guidelines, and preliminary data that are currently part of a separate report submitted for review showed that those cases that do not meet specific ATS/ERS/JRS/ ALAT diagnostic criteria had similar outcomes to those who did meet these diagnostic criteria.

In conclusion, we report a large national IPF cohort with outcome data extending up to 4 years. Patients from the AIPFR are diverse with a broad range of age, severity of lung function impairment and co-morbidities. Baseline lung function, GAP stage and PROMs were strong prognostic indicators in our cohort, and those on anti-fibrotic therapy did better independent of their underlying disease severity. These data support the crucial role that large longitudinal registries such as the AIPFR can play in better understanding the spectrum, natural history and clinical management of IPF in a real-world setting.

\section{Acknowledgements}

We would like to thank all the participants and physicians who contribute to the registry, as well as the registry secretariat and co-coordinators (Heather Chaplin, Sarah Richards, Karen Symons, Robert Henson, Susan Smith, Svetlana Baltic and Chris Emery) who work tirelessly to gather and collate information. We also thank Alfred Health Solutions and Alfred Medical Imaging, who are integral to the development and maintenance of the web-based radiology database.

\section{References}

1 Raghu G, Collard HR, Egan JJ, et al. An official ATS/ERS/JRS/ALAT statement: idiopathic pulmonary fibrosis: evidence-based guidelines for diagnosis and management. Am J Respir Crit Care Med 2011; 183: 788-824.

2 Richeldi L, du Bois RM, Raghu G, et al. Efficacy and safety of nintedanib in idiopathic pulmonary fibrosis. $N$ Engl J Med 2014; 370: 2071-2082.

3 King TE Jr, Bradford WZ, Castro-Bernardini S, et al. A phase 3 trial of pirfenidone in patients with idiopathic pulmonary fibrosis. N Engl J Med 2014; 370: 2083-2092.

4 Noble PW, Albera C, Bradford WZ, et al. Pirfenidone in patients with idiopathic pulmonary fibrosis (CAPACITY): two randomised trials. Lancet 2011; 377: 1760-1769.

5 Richeldi L, Costabel U, Selman M, et al. Efficacy of a tyrosine kinase inhibitor in idiopathic pulmonary fibrosis. N Engl J Med 2011; 365: 1079-1087.

6 Ley B, Collard HR, King TE Jr. Clinical course and prediction of survival in idiopathic pulmonary fibrosis. Am J Respir Crit Care Med 2011; 183: 431-440.

$7 \quad$ King TE Jr, Tooze JA, Schwarz MI, et al. Predicting survival in idiopathic pulmonary fibrosis: scoring system and survival model. Am J Respir Crit Care Med 2001; 164: 1171-1181.

8 Erbes R, Schaberg T, Loddenkemper R. Lung function tests in patients with idiopathic pulmonary fibrosis. Are they helpful for predicting outcome? Chest 1997; 111: 51-57.

9 Nadrous HF, Pellikka PA, Krowka MJ, et al. Pulmonary hypertension in patients with idiopathic pulmonary fibrosis. Chest 2005; 128: 2393-2399.

10 Nadrous HF, Myers JL, Decker PA, et al. Idiopathic pulmonary fibrosis in patients younger than 50 years. Mayo Clin Proc 2005; 80: 37-40.

11 Manali ED, Stathopoulos GT, Kollintza A, et al. The Medical Research Council chronic dyspnea score predicts the survival of patients with idiopathic pulmonary fibrosis. Respir Med 2008; 102: 586-592.

12 Iwasawa T, Asakura A, Sakai F, et al. Assessment of prognosis of patients with idiopathic pulmonary fibrosis by computer-aided analysis of CT images. J Thorac Imaging 2009; 24: 216-222.

13 Lettieri CJ, Nathan SD, Browning RF, et al. The distance-saturation product predicts mortality in idiopathic pulmonary fibrosis. Respir Med 2006; 100: 1734-1741.

14 Ley B, Bradford WZ, Vittinghoff E, et al. Predictors of mortality poorly predict common measures of disease progression in idiopathic pulmonary fibrosis. Am J Respir Crit Care Med 2016; 194: 711-718.

15 Ryerson CJ, Corte TJ, Collard HR, et al. A global registry for idiopathic pulmonary fibrosis: the time is now. Eur Respir J 2014; 44: 273-276.

16 Behr J, Kreuter M, Hoeper MM, et al. Management of patients with idiopathic pulmonary fibrosis in clinical practice: the INSIGHTS-IPF registry. Eur Respir J 2015; 46: 186-196.

17 Moodley Y, Goh N, Glaspole I, et al. Australian Idiopathic Pulmonary Fibrosis Registry: vital lessons from a national prospective collaborative project. Respirology 2014; 19: 1088-1091.

18 Ley B, Ryerson CJ, Vittinghoff E, et al. A multidimensional index and staging system for idiopathic pulmonary fibrosis. Ann Intern Med 2012; 156: 684-691.

19 Kaunisto J, Kelloniemi K, Sutinen E, et al. Re-evaluation of diagnostic parameters is crucial for obtaining accurate data on idiopathic pulmonary fibrosis. BMC Pulm Med 2015; 15: 92.

20 Ferrara G, Carlson L, Palm A, et al. Idiopathic pulmonary fibrosis in Sweden: report from the first year of activity of the Swedish IPF-Registry. Eur Clin Respir J 2016; 3: 31090.

21 Wells AU, Desai SR, Rubens MB, et al. Idiopathic pulmonary fibrosis: a composite physiologic index derived from disease extent observed by computed tomography. Am J Respir Crit Care Med 2003; 167: 962-969.

22 Raghu G, Rochwerg B, Zhang Y, et al. An official ATS/ERS/JRS/ALAT Clinical Practice Guideline: treatment of idiopathic pulmonary fibrosis. An update of the 2011 Clinical Practice Guideline. Am J Respir Crit Care Med 2015; 192: e3-e19.

23 O'Brien EC, Durheim MT, Gamerman V, et al. Rationale for and design of the Idiopathic Pulmonary Fibrosis-PRospective Outcomes (IPF-PRO) registry. BMJ Open Respir Res 2016; 3: e000108.

24 Hamada K, Nagai S, Tanaka S, et al. Significance of pulmonary arterial pressure and diffusion capacity of the lung as prognosticator in patients with idiopathic pulmonary fibrosis. Chest 2007; 131: 650-656.

25 Mogulkoc N, Brutsche MH, Bishop PW, et al. Pulmonary function in idiopathic pulmonary fibrosis and referral for lung transplantation. Am J Respir Crit Care Med 2001; 164: 103-108. 
26 Lynch DA, Godwin JD, Safrin S, et al. High-resolution computed tomography in idiopathic pulmonary fibrosis: diagnosis and prognosis. Am J Respir Crit Care Med 2005; 172: 488-493.

27 Shin KM, Lee KS, Chung MP, et al. Prognostic determinants among clinical, thin-section CT, and histopathologic findings for fibrotic idiopathic interstitial pneumonias: tertiary hospital study. Radiology 2008; 249: 328-337.

28 American Thoracic Society. Idiopathic pulmonary fibrosis: diagnosis and treatment. International consensus statement. American Thoracic Society (ATS), and the European Respiratory Society (ERS). Am J Respir Crit Care Med 2000; 161(2 Pt 1): 646-664.

29 Demedts M, Behr J, Buhl R, et al. High-dose acetylcysteine in idiopathic pulmonary fibrosis. N Engl J Med 2005; 353: 2229-2242.

30 Idiopathic Pulmonary Fibrosis Clinical Research Network, Raghu G, Anstrom KJ, et al. Prednisone, azathioprine, and N-acetylcysteine for pulmonary fibrosis. N Engl J Med 2012; 366: 1968-1977. 